\title{
Comparison of Spatio-Spectral Properties of Zen-Meditation and Resting EEG Based on Unsupervised Learning
}

\author{
Pei-Chen Lo*, Nasir Hussain \\ Institute of Electrical and Control Engineering, College of Electrical and Computer Engineering, \\ National Yang Ming Chiao Tung University, Taiwan \\ Email: *pclo756@g2.nctu.edu.tw
}

How to cite this paper: Lo, P.-C. and Hussain, N. (2021) Comparison of Spatio-Spectral Properties of Zen-Meditation and Resting EEG Based on Unsupervised Learning. Journal of Behavioral and Brain Science, 11, 58-72.

https://doi.org/10.4236/jbbs.2021.112005

Received: December 25, 2020

Accepted: February 22, 2021

Published: February 25, 2021

Copyright ( 2021 by author(s) and Scientific Research Publishing Inc. This work is licensed under the Creative Commons Attribution International License (CC BY 4.0).

http://creativecommons.org/licenses/by/4.0/

\begin{abstract}
This paper reports distinct spatio-spectral properties of Zen-meditation EEG (electroencephalograph), compared with resting EEG, by implementing unsupervised machine learning scheme in clustering the brain mappings of centroid frequency $(\mathrm{BMFc})$. Zen practitioners simultaneously concentrate on the third ventricle, hypothalamus and corpora quadrigemina touniversalize all brain neurons to construct a detached brain and gradually change the normal brain traits, leading to the process of brain-neuroplasticity. During such tri-aperture concentration, EEG exhibits prominent diffuse high-frequency oscillations. Unsupervised self-organizing map ( $\mathrm{SOM}$ ), clusters the dataset of quantitative EEG by matching the input feature vector $\mathrm{Fc}$ and the output cluster center through the SOM network weights. Input dataset contains brain mappings of 30 centroid frequencies extracted from CWT (continuous wavelet transform) coefficients. According to SOM clustering results, resting EEG is dominated by global low-frequency $(<14 \mathrm{~Hz})$ activities, except channels T7, F7 and TP7 $(>14.4 \mathrm{~Hz})$; whereas Zen-meditation EEG exhibits globally high-frequency $(>16$ $\mathrm{Hz}$ ) activities throughout the entire record. Beta waves with a wide range of frequencies are often associated with active concentration. Nonetheless, clinic report discloses that benzodiazepines, medication treatment for anxiety, insomnia and panic attacks to relieve mind/body stress, often induce beta buzz. We may hypothesize that Zen-meditation practitioners attain the unique state of mindfulness concentration under optimal body-mind relaxation.
\end{abstract}

\section{Keywords}

Electroencephalograph (EEG), Continuous Wavelet Transform (CWT), Unsupervised Learning, Self-Organizing Map (SOM), Spatio-Spectral Property, Zen Meditation 


\section{Introduction}

Even with the advanced imaging technologies in the scope of brain research, EEG (electroencephalograph) has been still favorable due to its superior temporal resolution of brain electrical activities. For decades, the electrical activities of human brain, recorded in the form of multi-channel waveforms, have been extensively studied in order to help clinicians diagnose and treat brain dysfunctions. More scientific evidence of EEG variations in correlation with diseases and pathologies has been disclosed, including apoplexy [1], epilepsy [2], encephalitis [3], aging [4] and epidemic diseases [5]. In addition, EEG studies have been employed in a wide scope of studies on cognitive functions [6], psychology [7], social behavior [8] and even body-mind practices including Yoga, Tai Chi, Qi Gong and meditation [9] [10]. Our previous study reported novel findings on EEG under Zen meditation, the ancient oriental practice of Zen sect aiming to attain the state of enlightenment [11] [12] [13]. This paper presents an innovative scheme, based on unsupervised deep learning, for analyzing spatio-spectral properties of resting and Zen-meditation EEG.

Mental exhaustion caused by overworked brain becomes common nowadays that is ascribed to the heavy information-processing demands. Zen meditation accordingly offers an effective way to relieve physiological and mental stress. In comparison with eyes-closed, relaxed rest, Zen meditation via tri-aperture concentration [14] effectively calms the brain and mind for bodily rejuvenation, according to the practitioners' experiences, differing from those worldwide popular meditation practices such as transcendental meditation (TM) from India, Japanese Zen, and Tibetan Buddhism [15]. Zen meditation follows the heartdominating principle while other meditations are mostly mind-cultivating practices. By concentrating on unique apertures, practitioners initiate the process of cleansing all conscious states in preparation for entering the realm of true nature deeply in the heart. Such process involves the functioning alteration of major components in the brain leading to brain plasticity. Brain function under such an undisclosed realm has drawn the attention of researchers. Our study has been focused on the EEG variations.

EEG is composed of waves of different frequencies ranging from $\delta(0-4 \mathrm{~Hz})$ to $\gamma(30-50 \mathrm{~Hz})$, associated with different physiological, pathological, psychological, mental or conscious states. For example, high alpha waves $(10-12 \mathrm{~Hz})$ emerge in the occipital region when a person is awake and relaxed with eyes closed [16] [17]. Spatial distribution of various EEG rhythms provides important indications of brain functioning. It is the first attempt to employ brain mapping of centroid frequency (BMFc) to characterize the spatio-spectral properties of multi-channel EEG from which the decomposed CWT [18] [19] coefficients are used to estimate the centroid frequency for each channel. To deal with the unknown complicated composition of BMFc dataset, self-organizing map (SOM) is implemented to classify and label the BMFc. SOM is a type of unsupervised learning method based on competitive learning. The output neurons of the net- 
work compete among themselves for the final decision of the one to be activated or fired. SOM (also named as Kohonen network) was pioneered by the Finnish researcher Teuvo Kohonen in 1980s [20] [21]. Since then, SOM has been extensively used as a clustering and visualization tool in data analysis. Differing from classical neural networks, SOM does not have any hidden layer. Based on the unsupervised learning scheme, the output neurons in SOM require no designated output values. Instead, each output neuron is identified by a vector of weights. SOM maps the high-dimensional space of input dataset to a lower dimensional output space with the original topological properties well preserved. The after-trained weight matrix thus enables an easier interpretation of complex inherent structures in the data. Applications of SOM range from pattern recognition [22] [23] to feature classification [24]. Through SOM classification, the cluster properties of BMFc represent the state of brain function associated with the multi-channel EEG that assist in the long-term EEG interpretation or clinical diagnosis.

\section{Methods}

\subsection{EEG Signals}

EEG signals were collected in 2002-2007 from 15 Zen-meditation practitioners (experimental group) with an average of 5.8-year meditation experience and 15 ordinary, healthy volunteers (control group). The average age of experimental group was 28 years, which was 23 years in the control group. Experimental subjects practiced Zen-meditation while control subjects were at rest in normal sitting position, with eyes closed. In the experiment, we conducted overall 50-minute recording of EEG signals for both groups. EEG signals were recorded by Power Lab/16SP recording system, utilizing the 30-channel, common-reference (linked-mastoid MS1-MS2) electrode montage based on the international 10 - 20 system. Figure 1 illustrates the EEG recording montage of the 30 electrode locations. To explore local rhythmic behavior, 9 scalp regions are examined:

Frontal region (F): Fp1, Fp2.

Longitudinal Midline-frontal (LMF): $\mathrm{Fz}, \mathrm{FCz}, \mathrm{Cz}$.

Longitudinal Midline-posterior (LMP): $\mathrm{CPz}, \mathrm{Pz}, \mathrm{O} 2$.

Transversal Midline (TM): T7, C3, Cz, C4, T8.

Left-frontal (LF): F7, F3, FT7, FC3.

Right-frontal (RF): F4, F8, FC4, FT8.

Left-posterior (LP): TP7, CP3, P7, P3.

Right-posterior (RP): CP4, TP8, P4, P8.

Occipital Lobe (O): O1, O2, Oz.

With the EEG electrodes circled in Figure 1.

The EEG signals were sampled at $200 \mathrm{~Hz}$. To remove the slow baseline drift and high-frequency artifacts, EEG were filtered by a band-pass filter with the passband of $0.5-50 \mathrm{~Hz}$. The segments seriously contaminated by such artifacts 


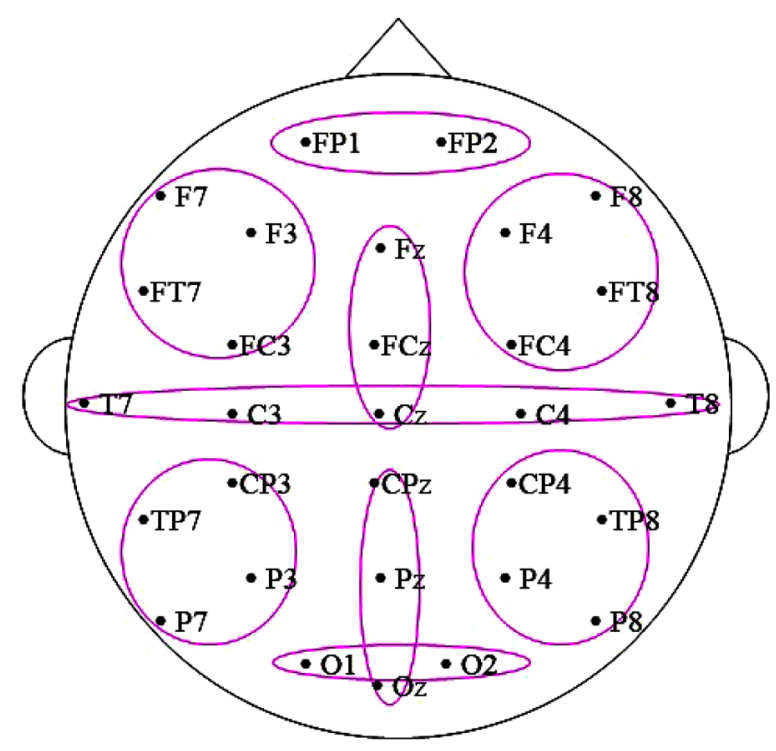

Figure 1. EEG recording montage (30-channel, using linked-mastoid MS1-MS2 as the common reference). Nine groups of electrodes are circled.

as eye blinking, eyeball movement and muscle activities were removed by naked-eye screening in the pre-processing stage.

\subsection{Construction of Centroid Frequency Brain Mapping (BMFc)}

Continuous wavelet transform (CWT) enables the optimization of time-frequency resolution, originally proposed in the milestone studies on multi-scale, multi-resolution processing [25] [26] [27]. The capability of time-frequency localization offers a way of extracting features at various locations in time at different scales. Time-frequency representation enables the exploration of local, transient or intermittent components. Let $C(a, b)$ denote the wavelet transform of a continuous time signal, $x(t)$, on the basis of the scaled (by a) and shifted (by $b$ ) wavelet $\psi_{a, b}(t)$. Wavelet transform is defined as the inner product of $x(t)$ and the complex conjugate $\psi_{a, b}^{*}(t)$,

$$
C(a, b)=\left\langle x(t), \psi_{a, b}(t)\right\rangle=\int_{-\infty}^{+\infty} x(t) \psi_{a, b}^{*}(t) \mathrm{d} t
$$

where $\psi(t)$ is the mother wavelet, or wavelet prototype. The child wavelet, $\psi_{a, b}(t)$, is derived from the mother wavelet $\psi(t)$ by,

$$
\psi_{a, b}(t)=\frac{1}{\sqrt{a}} \psi\left(\frac{t-b}{a}\right)
$$

Scale $a$ of the child waveletcorresponding to the frequency can be converted to the so-called pseudofrequency $f_{a}$ (in $\mathrm{Hz}$ ) by,

$$
f_{a}=\frac{f_{c}}{a \cdot f_{s}}
$$

where $f_{s}$ is the sampling period (second) and $f_{c}$ is the center frequency of a wavelet $(\mathrm{Hz})$ obtained from the frequency of the maximum magnitude of the Fourier 
transform of the wavelet. Our previous study [12] revealed that mother wavelet Daubechies 6 [28] is more feasible for EEG analysis. The family of Daubechies wavelets is known for its orthogonal property and efficient implementation.

$\mathrm{BMFc}$, brain mapping of centroid frequency, is constructed by Matlab toolbox EEGLAB developed by Swartz Center for Computational Neuroscience. Centroid frequency $F_{c}$ of EEG at a given time instant is computed by CWT coefficients $C_{f_{i}}$ in the frequency range of interest, $f_{L} \leq f_{i} \leq f_{U}$ (normally, $f_{U}=f_{s} / 2$, half the sampling rate).

$$
F_{c}=\frac{\sum_{f_{i}=f_{L}}^{f_{U}} f_{i} \cdot C_{f_{i}}}{\sum_{f_{i}=f_{L}}^{f_{U}} C_{f_{i}}}
$$

In this study, frequency range is $0.05 \mathrm{~Hz}-100 \mathrm{~Hz}$, with frequency resolution of $0.05 \mathrm{~Hz}$.

\subsection{Self-Organizing Map}

SOM applies competitive learning scheme to perform unsupervised training. Output neurons of the network compete among themselves for the final decision of the one to be activated or fired. Training of SOM network is aimed to find the $N_{o}$ vectors of weights characterizing the output clusters. Figure 2(a) displays the schematic architecture of 12-neuron SOM network.

Input dataset consists of $N_{i}$ feature vectors, $\xi_{i}=\left(x_{i 1}, x_{i 2}, \cdots, x_{i n_{d}}\right)$ for $1 \leq i \leq N_{i}$, where $n_{d}$ is the dimension of input vector. Hence, each vector is composed of $n_{d}$ centroid frequencies. Weight vector $\omega_{i j}\left(1 \leq i \leq N_{o}\right.$ and $1 \leq j \leq n_{d}$ ) connects the $f^{\text {th }}$ input element to the $i^{\text {th }}$ output neuron $o_{i}$. Output

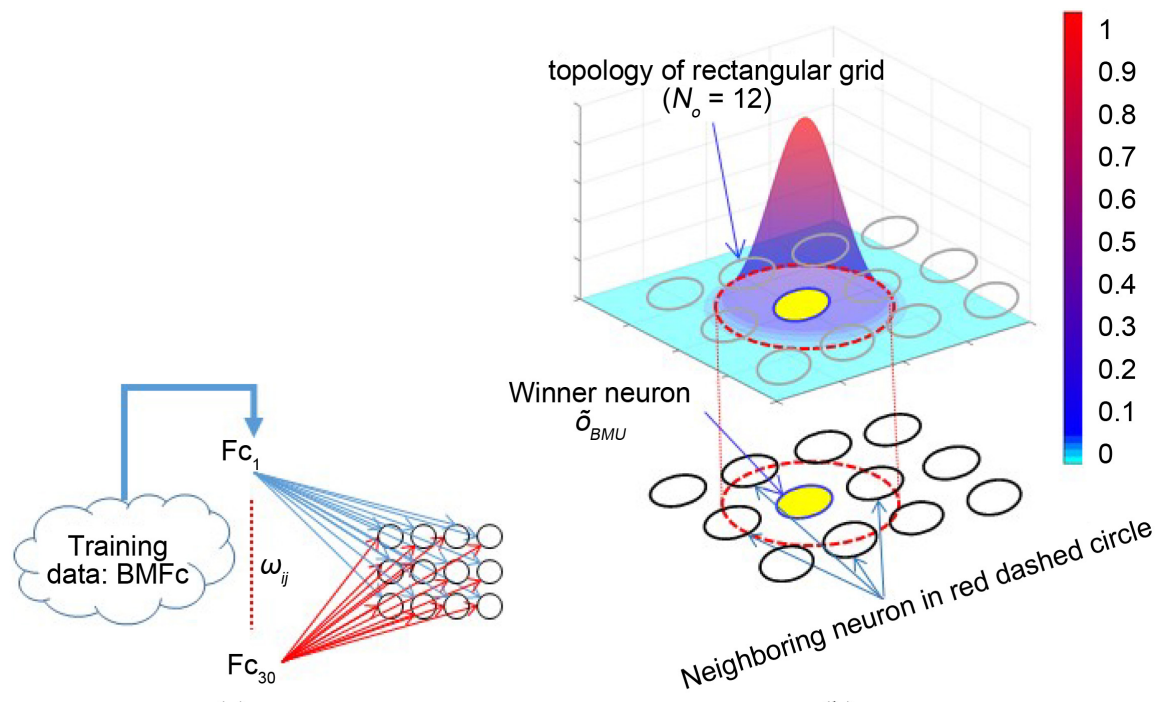

(a)

(b)

Figure 2. (a) Schematic architecture of 12-neuron SOM network. (b) Gaussian neighborhood function for determining various strengths of stimulation of the surrounding neurons. 
neuron $o_{i}$ is quantified by weight vector $\omega_{i}=\left\{\omega_{i j}, 1 \leq j \leq n_{d}\right\}$ for $i=1, \cdots, N_{o}$.In order to learn the input attributes without pre-labeled data, all the output neurons compete among themselves by searching for the weight vector $\omega_{j}$ that is most similar to the current input vector. Then output neuron $\tilde{o}_{j}$ will be declared as the winner neuron, also called the best matching unit (BMU). To find the BMU, distance between the current input vector $\boldsymbol{x}_{i}$ and the weight vectors $\boldsymbol{\omega}_{p}$ for $1 \leq j \leq N_{o}$ is computed by:

$$
D\left(\boldsymbol{x}_{i}, \boldsymbol{\omega}_{j}\right)=\left\|\boldsymbol{x}_{i}-\boldsymbol{\omega}_{j}\right\|, 1 \leq j \leq N_{o}
$$

$\mathrm{BMU}$ is the output neuron with the smallest distance:

$$
\tilde{o}_{\mathrm{BMU}}=\left\{\tilde{o}_{j} \mid \min _{j} D\left(\boldsymbol{x}_{i}, \boldsymbol{\omega}_{j}\right), j=1, \cdots, N_{o}\right\}
$$

BMU, acting as a firing neuron, stimulates more on the neurons closer to itself so that it produces various strengths of stimulation of the surrounding neurons (Figure 2(b)). Such a design is based on neurobiological evidence of lateral interaction among a set of excited neurons in the human brain. Once the $\tilde{o}_{\mathrm{BMU}}$ is determined, the weights (at the $i_{s}^{\text {th }}$ training step) are adjusted to approach the input vector $\boldsymbol{x}_{i}$ by [29].

$$
\omega_{j} \leftarrow \omega_{j}+\alpha\left(i_{s}\right) h_{j, B}\left(i_{s}\right)\left(x_{i}-\omega_{j}\right), 1 \leq j \leq N_{o}, 1 \leq i<N_{i}, 1 \leq i_{s}<N_{t}
$$

Input vectors are randomly fed into SOM $N_{t}$ times to ensure reshuffling of learning sequences. Weight-updating formula in (7) involves learning rate function $\alpha\left(i_{s}\right)$ and neighborhood function $h_{j, B}\left(i_{s}\right)$ :

$$
\begin{gathered}
\alpha\left(i_{s}\right)=\alpha_{0} \mathrm{e}^{-\frac{i_{s}}{N_{t}}}, 1 \leq i_{s} \leq N_{t} \\
h_{j, B}\left(i_{s}\right)=\mathrm{e}^{\frac{-\left\|r_{j}-r_{B}\right\|^{2}}{2 \sigma^{2}\left(i_{s}\right)}}, 1 \leq i_{s} \leq N_{t}
\end{gathered}
$$

where $\alpha_{0}$ is initial learning rate and $\left\|r_{j}-r_{B}\right\|$ is the lateral distance between $\tilde{o}_{\mathrm{BMU}}$ and the $f^{\text {th }}$ neuron in the grid of output neurons [29]. Learning rate function $\alpha\left(i_{s}\right)$ represents the rate of weight tuning and $\alpha\left(i_{s}\right)$ gradually decreases with the training time. Neighborhood function $h_{j, B}\left(i_{s}\right)$ is a two-dimensional Gaussian function (Figure 2(b)), with the purpose of producing more influence on weight update for neighboring neurons closer to $\tilde{O}_{\mathrm{BMU}}$. Gaussian standard deviation $\sigma\left(i_{s}\right)$ controls the progressive size of the neighborhood function at the $i_{s}^{\text {th }}$ training step that is an exponential decay function,

$$
\sigma\left(i_{s}\right)=\sigma_{0} \mathrm{e}^{-\frac{i_{s}}{1000 / \log \sigma_{0}}}, 1 \leq i_{s} \leq N_{t}
$$

where $\sigma_{0}$ is the initial neighborhood size.

To quantify the SOM performance, intra-cluster distance and inter-cluster distance are evaluated. Through exhaustive trials and tests, optimal parameters for SOM implementation may be determined by minimizing intra-cluster distance but maximizing inter-cluster distance, which are $N_{o}=12, N_{t}=10,000, \sigma_{0}=$ 3 and $\alpha_{0}=0.02$. 


\subsection{Evaluation of Clustering Performance}

The objective of data clustering is to identify various clusters within the dataset such that data with similar attributes are likely to be classified into the same cluster. Each cluster can be characterized by its cluster center that provides a medium for data visualization. Cluster center $\boldsymbol{c}_{i}$ is calculated by the average of all the elements (BMFc's) in cluster $\mathrm{C}_{i}$.

$$
\boldsymbol{c}_{i}=\frac{1}{N c_{i}} \sum_{\boldsymbol{x}_{j} \in \mathrm{C}_{i}} \boldsymbol{x}_{j}, 1 \leq i \leq N_{o}
$$

where $N c_{i}$ is the number of elements in cluster $i$.

A good clustering should result in a high intra-cluster similarity yet large inter-cluster deviations. Intra-cluster similarity is measured by intra-cluster distance $\left(D_{\text {in }}\right)$,

$$
D_{\text {in }}=\frac{1}{N_{c_{i}}} \sum_{\boldsymbol{x}_{j} \in \mathrm{C}_{i}}\left\|\boldsymbol{x}_{j}-\boldsymbol{c}_{i}\right\|
$$

where $\|\cdot\|$ denotes the Euclidean distance. Inter-cluster distance measures the degree of distinction among clusters. Inter-cluster distance $D_{\mathrm{ex}}$ is the average of all the pairwise inter-cluster distance $D_{i j}$ between cluster $\mathrm{C}_{i}$ and $\mathrm{C}_{j}$ is the distance between cluster center $\boldsymbol{c}_{i}$ and $c_{p} D_{i j}=\left\|\boldsymbol{c}_{i}-c_{j}\right\|$.

\section{Results}

Unsupervised 12-neuron SOM is applied to 60,000 BMFc's constructed respectively from 5-minute Zen-meditation EEG and resting EEG to study the spatial property of centroid frequency.

\subsection{Property of Centroid Frequency}

Figure 3 displaysthe 5-minute time evolution of centroid frequency for all 30 channels using temporal resolution of one second. Different colors represent different frequencies ranging from 10 to $22 \mathrm{~Hz}$ (the range of $\mathrm{Fc}$ computed). Zen-meditation EEG (Figure 3(b)) apparently exhibits higher frequency than resting EEG (Figure 3(a)).

The 5-minute resting EEG is dominated by global high- $\alpha$ rhythms $(10-12 \mathrm{~Hz})$ mixed with intermediate low $\beta(>12-16 \mathrm{~Hz})$ activities; whereas Zen-meditation EEG contains a large amount of low- $\beta$ rhythms $(13-18 \mathrm{~Hz})$ mostlyin Fz, F3, $\mathrm{FC} 4, \mathrm{FCz}, \mathrm{C} 4, \mathrm{Cz}, \mathrm{CPz}, \mathrm{CP} 4, \mathrm{P} 4$ and $\mathrm{Pz}$. Higher frequency in the $\beta$ band $(>20$ $\mathrm{Hz}$ ) appears in $\mathrm{O} 1, \mathrm{O} 2, \mathrm{Oz}, \mathrm{T} 7, \mathrm{TP} 7, \mathrm{CP} 3, \mathrm{FT} 8, \mathrm{~T} 8$ and $\mathrm{F} 7$.

To observe the macroscopic behavior of local rhythmic properties, Figure 4 displays the time evolution of the 30-second average centroid frequency of each of the 9 scalp regions depicted in Figure 1 (dividing 30 EEG channels into 9 groups). Region notation tagged with "-R (-Z)" indicates the result for resting (Zen-meditation) EEG. Curves of Fc-R (thin lines) group tightly in the range $13.5-15 \mathrm{~Hz}$, yet curves of Fc-Z (thick lines) extend from $15.5 \mathrm{~Hz}$ (LMF region) to $19.5 \mathrm{~Hz}$ (O region). 


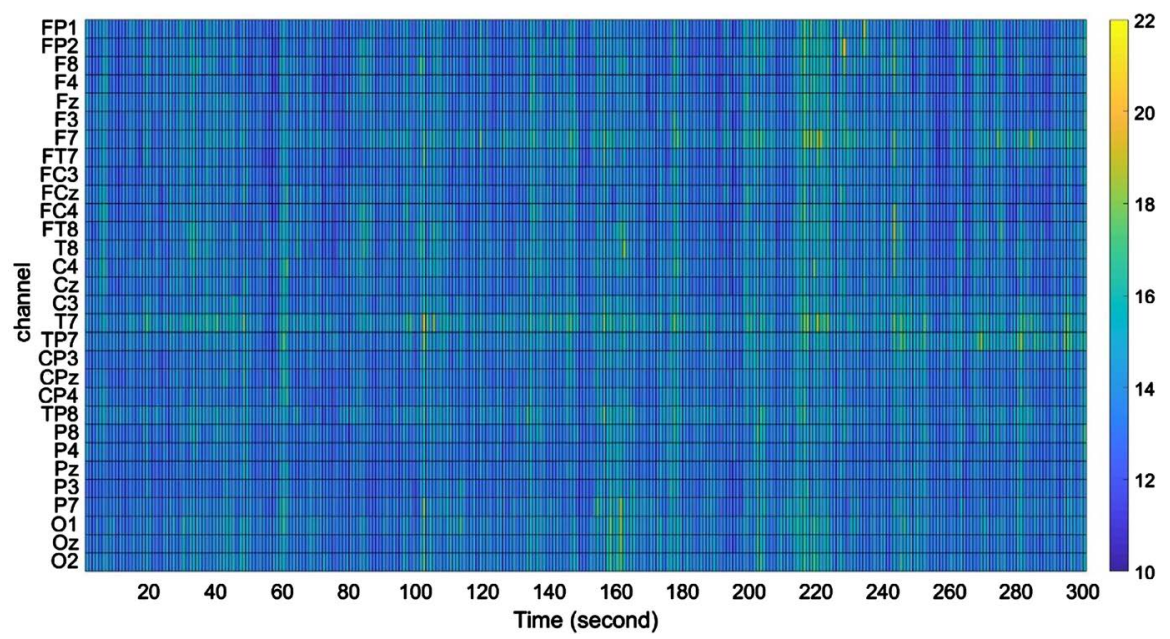

(a)

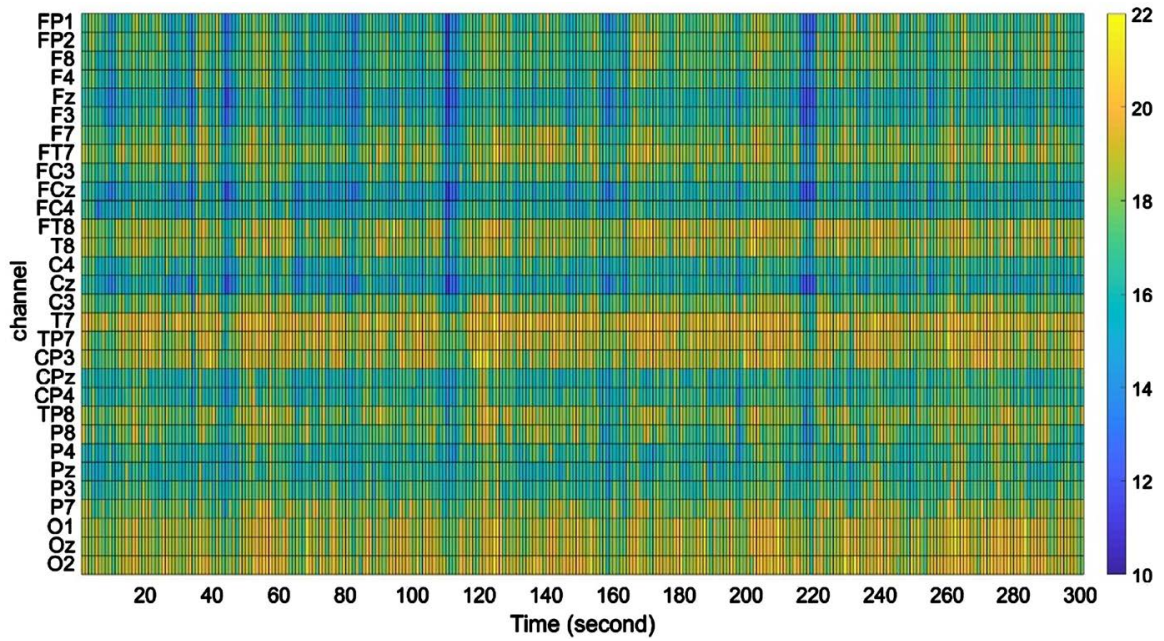

(b)

Figure 3. Time evolution of 30-channel centroid frequencies for (a) resting EEG, and (b) Zen-meditation EEG.

In resting EEG, faster activities (average $14.11 \mathrm{~Hz}$ ) appear in the TM region almost throughout the entire 5-minute duration; whereas slower EEG rhythms (average $13.71 \mathrm{~Hz}$ ) are observed in the LMP region. In Zen-meditation EEG, slower EEG activities $(15.5 \mathrm{~Hz})$ appear in the LMF region almost throughout the entire 5-minute duration; whereas faster EEG rhythms are observed mostly in the occipital region $(19.5 \mathrm{~Hz})$.

\subsection{Results of SOM Clustering}

Dataset of BMFC are classified by 12-neuron SOM using 10,000 training epochs $\left(N_{t s}\right)$, initial learning rate $(\alpha 0) 0.02$ and initial neighborhood size $(\sigma 0) 3$. Table 1 displays the BMFc's of 12 cluster centers of 12 cluster centers of 5-minute resting (upper) and Zen-meditation (lower) EEG. Each cluster center is computed by averaging all the cluster members inside the cluster. Average (std), highest and lowest frequency of each cluster center are listed under each BMFc. The cluster 


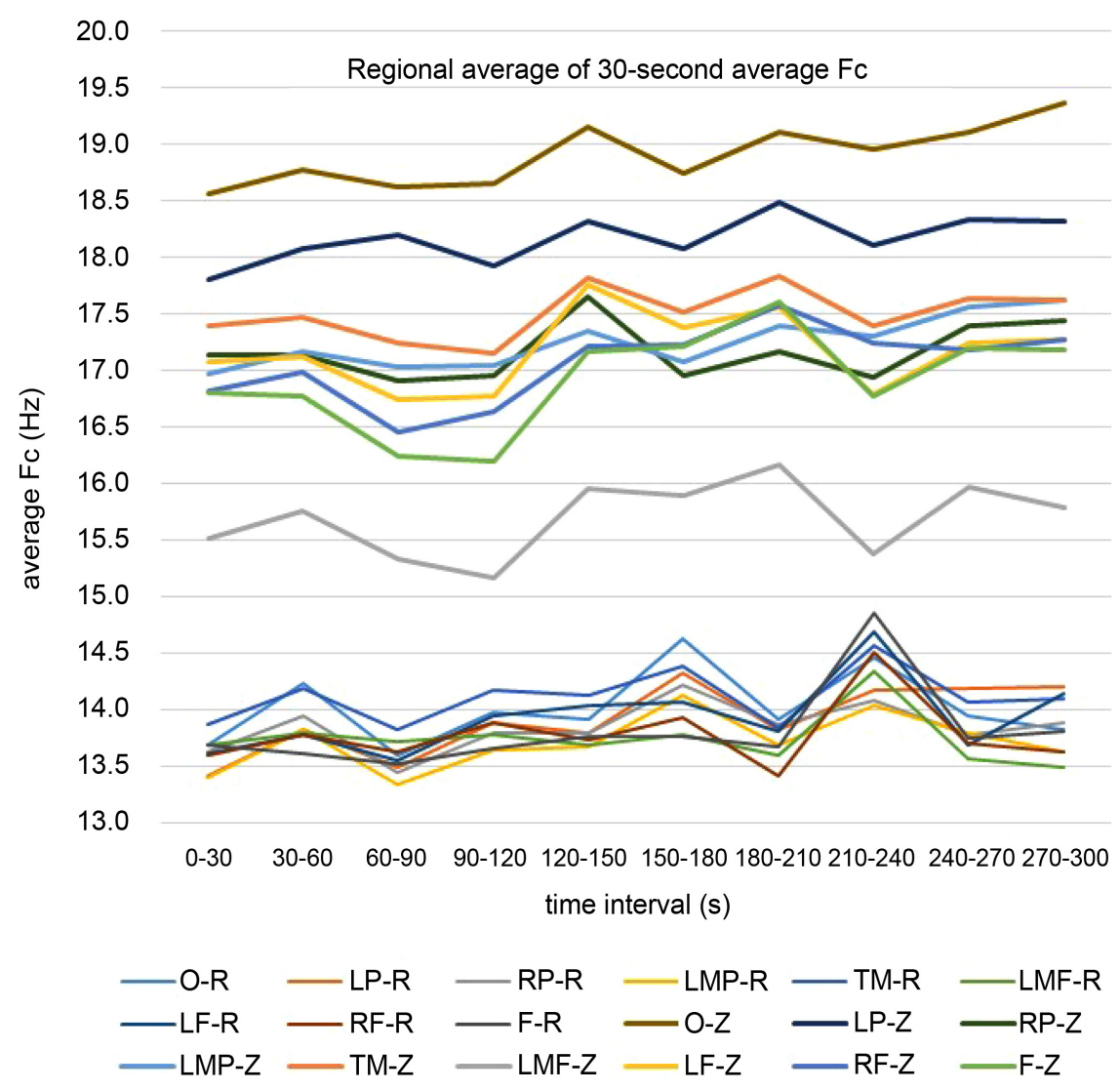

Figure 4. Regional average of 30-second average centroid frequency.

is indexed according to the cluster size so that $\mathrm{C} 1$ (C12) contains the most (fewest) elements.

Clustering result reveals more than $75 \%$ high-alpha $(10-12 \mathrm{~Hz})$ dominant BMFc's in resting EEG (average $\mathrm{Fc}<15 \mathrm{~Hz}$ in clusters $\mathrm{C} 1$ - C8), yet more than $80 \%$ beta dominant BMFs's in Zen-meditation EEG (minimum $\mathrm{Fc}>13 \mathrm{~Hz}$ in all clusters except $\mathrm{C} 2$ and $\mathrm{C} 11)$.Cluster-center $\mathrm{BMFc}$ exhibits the average intra-cluster characteristics of all the elements (BMFc's). To explore the detailed spatio-spectral activities, Table 2 displays the representative BMFc's randomly selected from top four clusters of resting and Zen-meditation EEG.

The top four clusters of BMFc of resting EEG are characterized by the following spatio-spectral properties:

C1: global high- $\alpha$ rhythms $(\sim 12 \mathrm{~Hz})$;

C2: mainly high $\alpha$ around $13 \mathrm{~Hz}$, with intermittent left-frontal low $\beta$ (13 - 14 $\mathrm{Hz}$ );

C3: global high $\alpha(\sim 13 \mathrm{~Hz})$ mixed withright-temporal low $\beta(15-16 \mathrm{~Hz})$;

C4: background high $\alpha(12-13 \mathrm{~Hz})$ with occipital localized low $\beta(15-16 \mathrm{~Hz})$.

The top four clusters of BMFc of Zen-meditation EEG exhibit quite different spatio-spectral scenarios:

C1: $14-17 \mathrm{~Hz}$ background mixed with intermittent high $\beta(20-25 \mathrm{~Hz})$, slower activities in posterior regions; 
$\mathrm{C} 2$ : similar to $\mathrm{C} 1$, yet slower rhythms in frontal regions and intermittent high $\beta$ activity $(\sim 22 \mathrm{~Hz})$ recurring in occipital area;

C3: $15-19 \mathrm{~Hz} \beta$ band, right-temporal higher $\beta(20-25 \mathrm{~Hz})$ extending to right occipital regions;

C4: similar to $\mathrm{C} 3$, yet high $\beta$ migrating towards left temporal regions.

Different clusters accordingly portray different spatio-spectral properties in the entire resting (Zen-meditation) course. Long-term multi-channel EEG

Table 1. BMFc's of 12 cluster centers of 5-minute resting (upper) and Zen-meditation (lower) EEG. Average (std), highest and lowest frequency of each cluster center are listed under each BMFc.

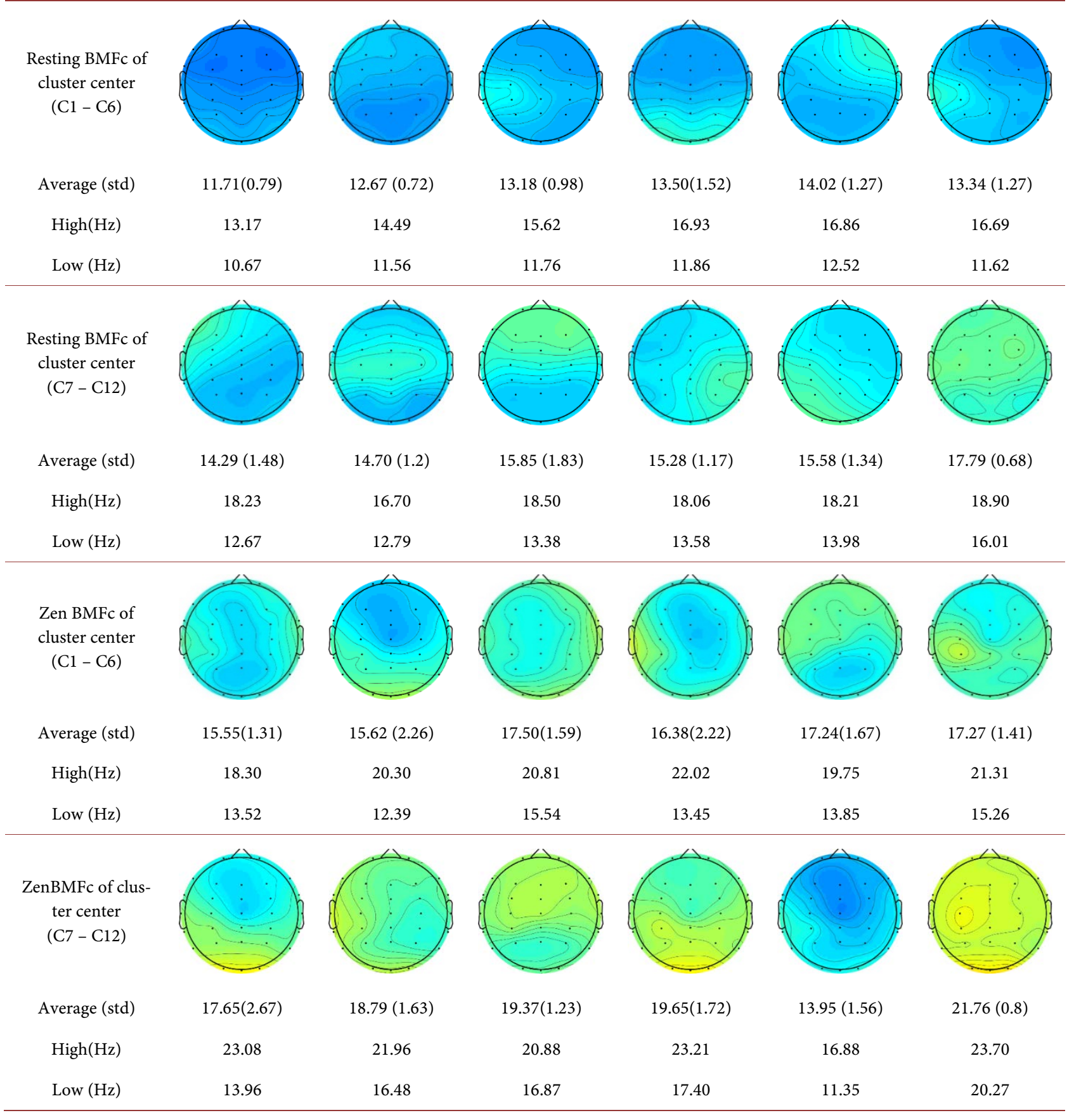


Table 2. Representative BMFc's randomly selected from top four clusters of resting and Zen-meditation EEG.

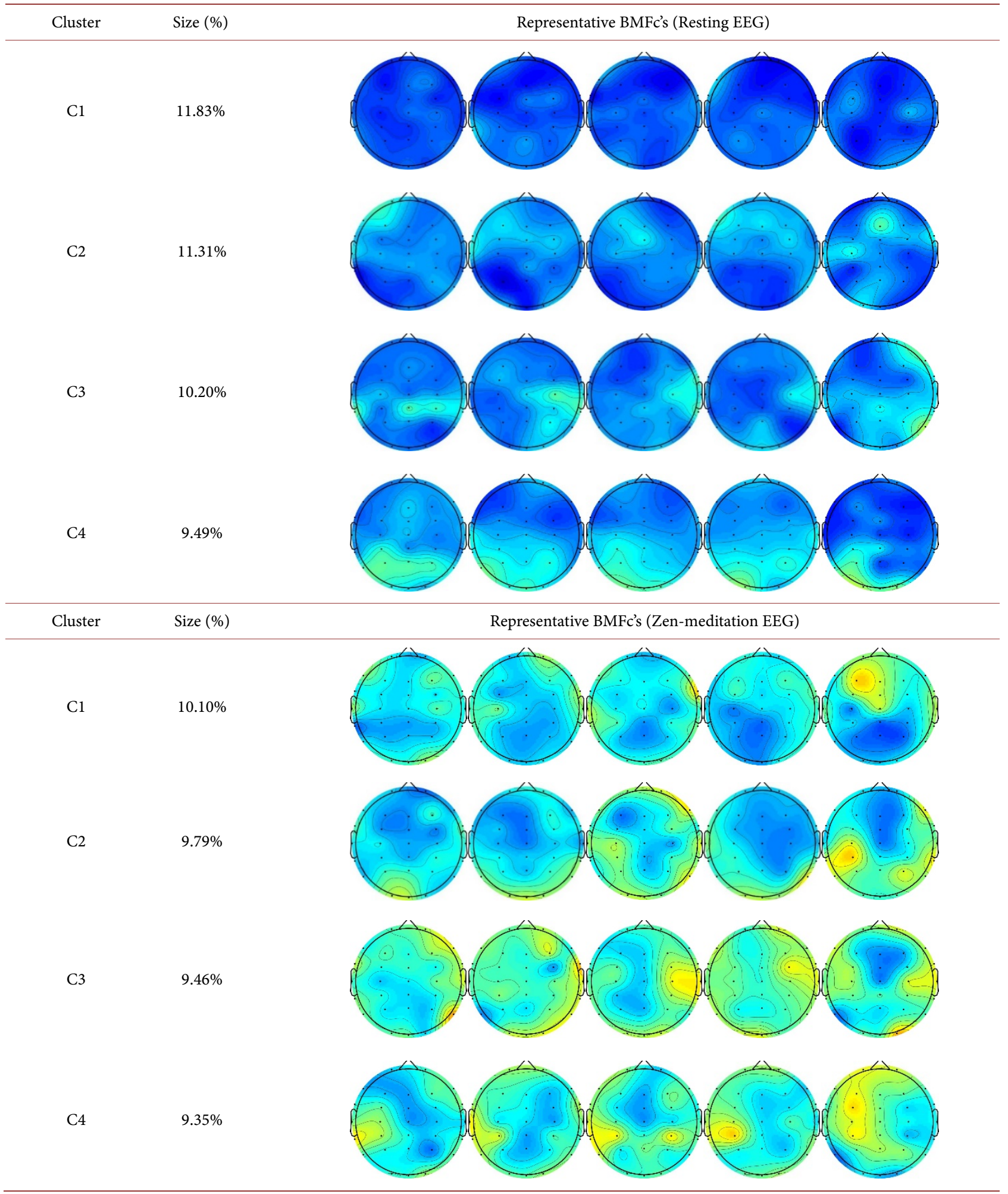

record may be interpreted by the cluster id that provides an alternative illustration of the temporal scenario of spatio-spectral characteristics. Figure 5 demonstrates the BMFc scenarios of (a) resting and (b) Zen-meditation EEG using a 


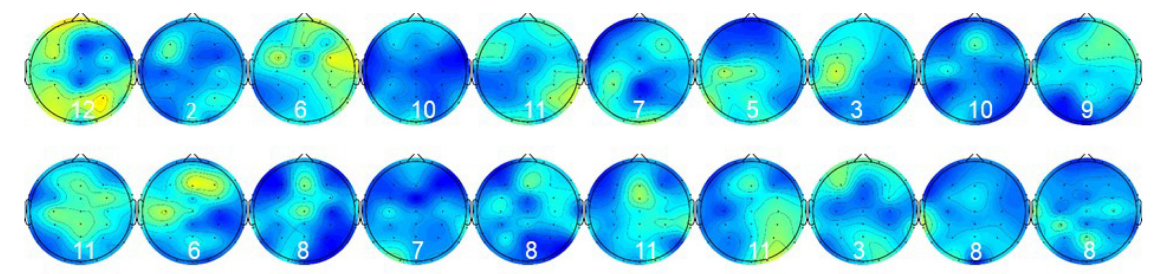

(a)

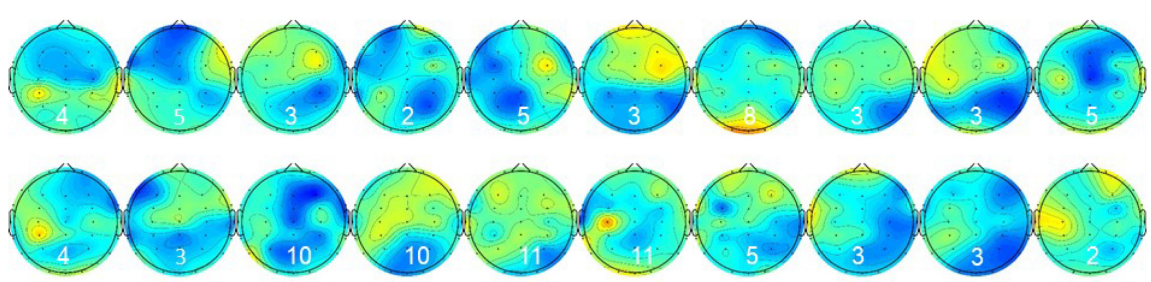

(b)

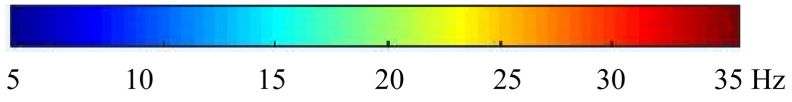

Figure 5. BMFc scenarios of (a) resting and (b) Zen-meditation EEG (moving step of 0.05 second), labeled by the cluster id at the bottom of each BMFc.

step size of 0.05 second, with the cluster id shown at the bottom of each BMFc.

Higher Fc of resting EEG mostly focalizes in the left inferior temporal regions. On the other hand, Zen meditation EEG exhibits faster rhythms in a larger scalp area, including left inferior temporal, occipital and frontal regions. Figure 6 displays the distribution of the 5-minute average centroid frequency at each electrode site for (left) resting and (right) Zen-meditation EEG, that well corroborates the spatial property mentioned.

\section{Conclusions and Discussions}

The main contribution of this study includes the finding of global rapid rhythms (beta band) in Zen-meditation EEG and the introduction of unsupervised, self-learning scheme to the analysis of abundant complicated biomedical data involving spatio-spectral variations. BMFc quantifying the EEG spatial-spectral properties exhibits prominent difference between resting and Zen-meditation EEG. Zen-meditation EEG oscillates at the rhythms 3 - $5 \mathrm{~Hz}$ higher than resting EEG all over the entire scalp. The maximum of average centroid frequency is $20.77 \mathrm{~Hz}$ and the minimum of average centroid frequency is $15.12 \mathrm{~Hz}$ for Zen-meditation EEG, which is respectively $14.75 \mathrm{~Hz}$ and $13.57 \mathrm{~Hz}$ for resting EEG. Beta rhythms in the mid-beta band $(16.5-20 \mathrm{~Hz})$ distribute almost all over the scalp except a small mid-central region $(\mathrm{Fz}, \mathrm{FCz}, \mathrm{Cz})$. Evidently, Zen-meditation EEG exhibits higher centroid frequency with a larger frequency range. Moreover, local spectral properties as shown by the centroid frequency of each individual channel reflect consistently faster rhythmic activities in Zen-meditation EEG than which in resting EEG. Beta rhythms have been associated with mind-body intervention, attention, emotion and cognition during 


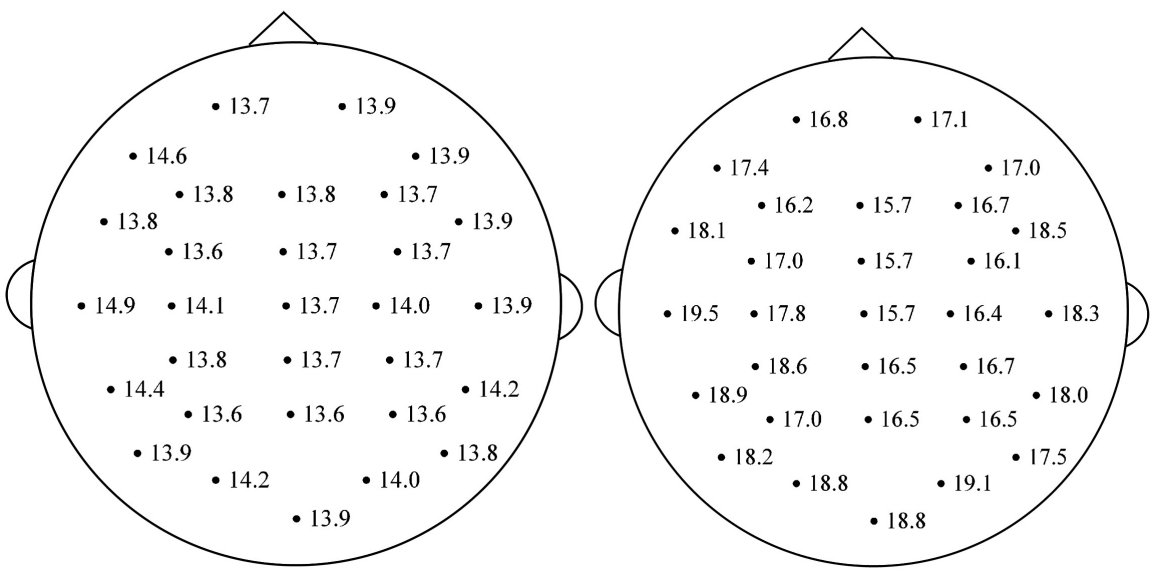

Figure 6. Distribution of 5-minute average centroid frequency for (left) resting and (right) Zen-meditation EEG.

the past decade [30]. Previous study reports the increase of occipital beta power in the attentional task with correct responses but lack of beta activity change before erroneous responses [31]. Global high beta rhythms, particularly at channels $\mathrm{Oz}, \mathrm{O} 1$ and $\mathrm{O} 2$, of Zen-meditation EEG imply the state of exceptional mindfulness concentration evoked by Zen meditation.

\section{Acknowledgements}

This research was supported by the grants from the Ministry of Science and Technology of Taiwan (Grant No.: MOST 108-2221-E-009-079). We are thankful to Master student Shao-Hong Wang for his support of computer programming and data analysis.

\section{Conflicts of Interest}

We have no conflicts of interests to disclose.

\section{References}

[1] Jordan, K.G. (2004) Emergency EEG and Continuous EEG Monitoring in Acute Ischemic Stroke. Journal of Clinical Neurophysiology, 21, 341-352.

[2] Srinivasan, V., Eswaran, C. and Sriraam, N. (2007) Approximate Entropy-Based Epileptic EEG Detection Using Artificial Neural Networks. IEEE Transactions on Information Technology in Biomedicine, 11, 288-295. https://doi.org/10.1109/TITB.2006.884369

[3] Kaplan, P.W., Rossetti, A.O., Kaplan, E.H. and Wieser, H.G. (2012) Proposition: Limbic Encephalitis May Represent Limbic Status Epilepticus. A Review of Clinical and EEG Characteristics. Epilepsy \& Behavior, 24, 1-6. https://doi.org/10.1016/j.yebeh.2011.11.029

[4] Koenig, T., Prichep, L., Dierks, T., Hubl, D., Wahlund, L.O., John, E.R. and Jelic, V. (2005) Decreased EEG Synchronization in Alzheimer's Disease and Mild Cognitive Impairment. Neurobiology of Aging, 26, 165-171. https://doi.org/10.1016/j.neurobiolaging.2004.03.008

[5] Canham, L.J.W., Staniaszek, L.E., Mortimer, A.M., Nouri, L.F. and Kane, N.M. 
(2020) Electroencephalographic (EEG) Features of Encephalopathy in the Setting of Covid-19: A Case Series. Clinical Neurophysiology Practice, 5, 199-205. https://doi.org/10.1016/j.cnp.2020.06.001

[6] Bell, M.A. and Cuevas, K. (2012) Using EEG to Study Cognitive Development: Issues and Practices. Journal of Cognition and Development, 13, 281-294. https://doi.org/10.1080/15248372.2012.691143

[7] Lin, M.H., Cross, S.N.N., Jones, W.J. and Childers, T.L. (2018) Applying EEG in Consumer Neuroscience. European Journal of Marketing, 52, 66-91. https://doi.org/10.1108/EJM-12-2016-0805

[8] Sutton, S.K., Burnette, C.P., Mundy, P.C., Meyer, J., Vaughan, A., Sanders, C. and Yale, M. (2005) Resting Cortical Brain Activity and Social Behavior in Higher Functioning Children with Autism. Journal of Child Psychology and Psychiatry, 46, 211-222. https://doi.org/10.1111/j.1469-7610.2004.00341.x

[9] Cahn, B.R. and Polich, J. (2006) Meditation States and Traits: EEG, ERP, and Neuroimaging Studies. Psychological Bulletin, 132, 180-211. https://doi.apa.org/doi/10.1037/0033-2909.132.2.180

[10] Lehmann, D., Faber, P.L., Tei, S., Pascual-Marqui, R.D., Milz, P. and Kochi, K. (2012) Reduced Functional Connectivity between Cortical Sources in Five Meditation Traditions Detected with Lagged Coherence Using EEG Tomography. Neuroimage, 60, 1574-1586. https://doi.org/10.1016/j.neuroimage.2012.01.042

[11] Lo, P.C., Huang, M.L. and Chang, K.M. (2003) EEG Alpha Blocking Correlated with Perception of Inner Light during Zen Meditation. The American Journal of Chinese Medicine, 31, 629-642. https://doi.org/10.1142/S0192415X03001272

[12] Lo, P.C. and Chang, C.H. (2013) Spatially Nonlinear Interdependence of Alpha-Oscillatory Neural Networks under Chan Meditation. Evidence-Based Complementary and Alternative Medicine, 2013, Article ID: 360371. https://doi.org/10.1155/2013/360371

[13] Lo, P.C., Tsai, P.H., Kang, H.J. and Wu, J.M.T. (2018) Cardiorespiratory and Autonomic-Nervous-System Functioning of Drug Abusers Treated by Zen Meditation. Journal of Traditional and Complementary Medicine, 9, 215-220. https://doi.org/10.1016/j.jtcme.2018.01.005

[14] Wu, J.M.T. (2010) Chan Master Miao Tian's Book of Wisdom and the Guide to Heart Chan Meditation. Lulu Press, USA.

[15] Kaur, C. and Singh, P. (2015) EEG Derived Neuronal Dynamics during Meditation: Progress and Challenges. Advances in Preventive Medicine, 2015, Article ID: 614723. https://doi.org/10.1155/2015/614723

[16] Lo, P.C. and Leu, J.S. (2005) Quantification of Pseudo-Periodicity of Alpha Rhythm in Meditation EEG. Journal of Medical and Biological Engineering, 25, 7-13.

[17] Chang, K.M. and Lo, P.C. (2006) F-VEP and Alpha-Suppressed EEG-Physiological Evidence of Inner-Light Perception during Zen Meditation. Biomedical Engineering. Applications, Basis and Communications, 18, 1-7. https://doi.org/10.4015/S1016237206000026

[18] Sadowsky, J. (1994) The Continuous Wavelet Transform: A Tool for Signal Analysis and Understanding. Johns Hopkins APL Technical Digest, 15, 306-318.

[19] Aguiar-Conraria, L. and Soares, M.J. (2013) The Continuous Wavelet Transform: Moving Beyond Uni- and Bivariate Analysis. Journal of Economic Surveys, 28, 344-375. https://doi.org/10.1111/joes.12012

[20] Kohonen, T., Oja, E., Simula, O., Visa, A. and Kangas, J. (1996) Engineering Appli- 
cations of the Self-Organizing Map. Proceedings of the IEEE, 84, 1358-1384. https://doi.org/10.1109/5.537105

[21] Vesanto, J. and Alhoniemi, E. (2000) Clustering of the Self-Organizing Map. IEEE Transactions on Neural Networks, 11, 586-600. https://doi.org/10.1109/72.846731

[22] Richardson, A.J., Risien, C. and Shillington, F.A. (2003) Using Self-Organizing Maps to Identify Patterns in Satellite Imagery. Progress in Oceanography, 59, 223-239. https://doi.org/10.1016/j.pocean.2003.07.006

[23] O'Connell, C., Kutics, A. and Nakagawa, A. (2013) Layered Self-Organizing Map for Image Classification in Unrestricted Domains. 2013 International Conference on Image Analysis and Processing, Naples, 9-13 September 2013, 310-319. https://doi.org/10.1007/978-3-642-41181-6_32

[24] Kohonen, T. (2013) Essentials of the Self-Organizing Map. Neural Networks, 37, 52-65. https://doi.org/10.1016/j.neunet.2012.09.018

[25] Daubechies, I. (1990) The Wavelet Transform, Time-Frequency Localization and Signal Analysis. IEEE Transactions on Information Theory, 36, 961-1005. https://doi.org/10.1109/18.57199

[26] Grossmann, A. and Morlet, J. (1984) Decomposition of Hardy Functions into Square Integrable Wavelets of Constant Shape. SIAM Journal on Mathematical Analysis, 15, 723-736. https://doi.org/10.1137/0515056

[27] Meyer, Y. (1992) Wavelets and Operators. Cambridge Studies in Advanced Math. Vol. 37, Cambridge University Press, Cambridge.

[28] Popov, D., Gapochkin, A. and Nekrasov, A. (2018) An Algorithm of Daubechies Wavelet Transform in the Final Field When Processing Speech Signals. Electronics, 7, 120. https://doi.org/10.3390/electronics7070120

[29] Lo, P.C. and Wu, Y.Z. (2019) Cross-Bifrequency Analysis of Cardiorespiratory Interactions at Rest and Zen Meditation. The 6 th International Conference on Systems and Informatics, Shanghai, 2-4 November 2019, 1117-1120. https://doi.org/10.1109/ICSAI48974.2019.9010252

[30] Lee, D.J., Kulubya, E., Goldin, P., Goodarzi, A. and Girgis, F. (2018) Review of the Neural Oscillations Underlying Meditation. Frontiers in Neuroscience, 12, 178. https://doi.org/10.3389/fnins.2018.00178

[31] Gola, M., Magnuski, M., Szumska, I. and Wróbel, A. (2013) EEG Beta Band Activity Is Related to Attention and Attentional Deficits in the Visual Performance of Elderly Subjects. International Journal of Psychophysiology, 89, 334-341. https://doi.org/10.1016/j.ijpsycho.2013.05.007

\section{List of Abbreviation}

ANN: artificial neural network;

BMFc: brain mapping of centroid frequencies;

CWT: continuous wavelet transforms;

SOM: self-organizing map. 